Louisiana State University

LSU Digital Commons

$5-19-2010$

\title{
Quantum criticality due to incipient phase separation in the two- dimensional Hubbard model
}

\author{
E. Khatami \\ Louisiana State University \\ K. Mikelsons \\ Louisiana State University \\ D. Galanakis \\ Louisiana State University
}

A. MacRidin

Fermi National Accelerator Laboratory

J. Moreno

Louisiana State University

See next page for additional authors

Follow this and additional works at: https://digitalcommons.Isu.edu/physics_astronomy_pubs

\section{Recommended Citation}

Khatami, E., Mikelsons, K., Galanakis, D., MacRidin, A., Moreno, J., Scalettar, R., \& Jarrell, M. (2010).

Quantum criticality due to incipient phase separation in the two-dimensional Hubbard model. Physical

Review B - Condensed Matter and Materials Physics, 81 (20) https://doi.org/10.1103/

PhysRevB.81.201101

This Article is brought to you for free and open access by the Department of Physics \& Astronomy at LSU Digital Commons. It has been accepted for inclusion in Faculty Publications by an authorized administrator of LSU Digital Commons. For more information, please contact ir@lsu.edu. 


\section{Authors}

E. Khatami, K. Mikelsons, D. Galanakis, A. MacRidin, J. Moreno, R. T. Scalettar, and M. Jarrell 


\section{San Jose State University}

From the SelectedWorks of Ehsan Khatami

May, 2010

\section{Quantum criticality due to incipient phase separation in the two-dimensional Hubbard model}

Ehsan Khatami, Louisiana State University

K. Mikelsons, University of Cincinnati

D. Galanakis, Louisiana State University

A. Macridin, Fermilab

J. Moreno, Louisiana State University, et al. 


\title{
Quantum criticality due to incipient phase separation in the two-dimensional Hubbard model
}

\author{
E. Khatami, ${ }^{1,2}$ K. Mikelsons, ${ }^{1,2}$ D. Galanakis, ${ }^{1}$ A. Macridin, ${ }^{3}$ J. Moreno, ${ }^{1}$ R. T. Scalettar, ${ }^{4}$ and M. Jarrell ${ }^{1}$ \\ ${ }^{1}$ Department of Physics and Astronomy, Louisiana State University, Baton Rouge, Louisiana 70803, USA \\ ${ }^{2}$ Department of Physics, University of Cincinnati, Cincinnati, Ohio 45221, USA \\ ${ }^{3}$ Fermilab, P.O. Box 500, Batavia, Illinois 60510, USA \\ ${ }^{4}$ Department of Physics, University of California, Davis, California 95616, USA
}

(Received 19 August 2009; revised manuscript received 11 April 2010; published 19 May 2010)

\begin{abstract}
We investigate the two-dimensional Hubbard model with next-nearest-neighbor hopping, $t^{\prime}$, using the dynamical cluster approximation. We confirm the existence of a first-order phase-separation transition terminating at a second-order critical point at filling $n_{c}\left(t^{\prime}\right)$ and temperature $T_{p s}\left(t^{\prime}\right)$. We find that as $t^{\prime}$ approaches zero, $T_{p s}\left(t^{\prime}\right)$ vanishes and $n_{c}\left(t^{\prime}\right)$ approaches the filling associated with the quantum critical point separating the Fermi liquid from the pseudogap phase. We propose that the quantum critical point under the superconducting dome is the zero-temperature limit of the line of second-order critical points.
\end{abstract}

DOI: 10.1103/PhysRevB.81.201101

PACS number(s): 71.10.Fd, 71.10.Hf, 74.72.- h

\section{INTRODUCTION}

Strongly correlated electronic materials, which include high-temperature superconductors, heavy fermions, and magnetic compounds, are characterized by competing phases and complicated phase diagrams. These competing phases can lead to quantum criticality when one of the transition temperatures is driven to absolute zero as a function of a nonthermal control parameter such as pressure or doping. ${ }^{1,2}$ While the physics of a conventional phase transition is driven by thermal fluctuations, near a quantum critical point (QCP), quantum fluctuations affect the properties of a material up to surprisingly high temperatures. ${ }^{3}$ In particular, transport measurements of hole-doped cuprates suggest the presence of a QCP (Refs. 4 and 5) lying beneath the superconducting (SC) dome. ${ }^{6,7}$ Although it is believed that this QCP dominates the phase diagram, its nature is still unknown ${ }^{8}$ with competing scenarios emphasizing the role of bosonic or fermionic fluctuations. ${ }^{4}$

In this work, we provide evidence for the nature of the QCP in the Hubbard model of the cuprates by a systematic study of its phase diagram. Our results suggest that the QCP is not due to order in the pseudogap (PG) region, but rather is the zero-temperature limit of a line of second-order critical points associated with a first-order phase-separation transition (see Fig. 1). The control parameter for this transition is the next-near-neighbor hopping parameter, $t^{\prime}$.

Although it is possible to have a QCP not associated with any obvious order parameter, as in the case of a localization transition, in most QCPs a continuous order parameter vanishes at $T=0$ for a particular value of the controlling energy scale. ${ }^{9}$ Less common is a QCP associated with a first-order transition, but this is possible when the first-order transition terminates at a second-order critical point which is driven to zero by tuning the relevant parameter. For example, in $\mathrm{Sr}_{3} \mathrm{Ru}_{2} \mathrm{O}_{7},{ }^{10}$ as a function of the field angle, a first-order metamagnetic transition is driven to $T=0$ yielding quantum critical phenomena.

The latter case is consistent with the scenario of Fig. 1, where the role of the field angle is played by $t^{\prime}$. For positive $t^{\prime}$ and below a certain temperature, the system undergoes a first-order phase-separation transition. In this region, two solutions with different densities coexist for a given chemical potential, $\mu .^{11,12}$ More recently, this first-order metalinsulator transition was studied by Gull et al. ${ }^{13}$ and used to map out the phase diagram of this model in the space of interaction strength and $t^{\prime}$. Since these two phases have the same symmetry, this transition terminated in a second-order critical point at temperature $T_{p s}$ and critical filling $n_{c}$ where the charge susceptibility diverges. By increasing $t^{\prime}, T_{p s}$ increases and the critical point becomes numerically accessible. For low $t^{\prime}$, it is no longer accessible, but its presence is evidenced by a peak in the charge susceptibility.

Our starting point is the two-dimensional (2D) Hubbard Hamiltonian

$$
H=\sum_{\mathbf{k} \sigma} \epsilon_{\mathbf{k}}^{0} c_{\mathbf{k} \sigma}^{\dagger} c_{\mathbf{k} \sigma}+U \sum_{i} n_{i \uparrow} n_{i \downarrow},
$$

where $\epsilon_{\mathbf{k}}^{0}=-2 t\left(\cos k_{x}+\cos k_{y}\right)-4 t^{\prime}\left(\cos k_{x} \cos k_{y}-1\right)$ is the tight-binding dispersion as a function of the hopping $t$ between nearest neighbors and $t^{\prime}$ between next-nearest neigh-

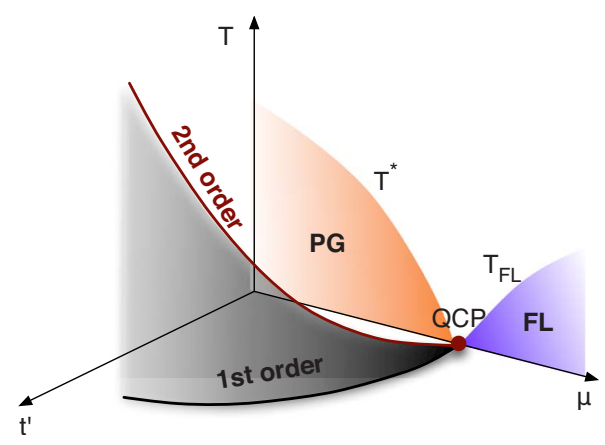

FIG. 1. (Color online) Schematic phase diagram of the 2D Hubbard model in the temperature $(T)$, chemical potential $(\mu)$, and nextnear-neighbor hopping $\left(t^{\prime}\right)$ space. For $t^{\prime}>0$ the first-order phaseseparation transition terminates at a second-order critical point at doping $n_{c}$ and temperature $T_{p s}$. The line of second-order critical points $\left(T_{p s}, n_{c}\right)$ approaches the QCP on the $t^{\prime}=0$ plane. This is the critical point separating the pseudogap (PG) from the Fermi-liquid (FL) region. 
bors, $c_{\mathbf{k} \sigma}^{\dagger}\left(c_{\mathbf{k} \sigma}\right)$ is the creation (annihilation) operator for electrons of wave vector $\mathbf{k}$ and $\operatorname{spin} \sigma, n_{i \sigma}=c_{i \sigma}^{\dagger} c_{i \sigma}$, and $U$ is the on-site Coulomb repulsion.

\section{METHODOLOGY}

We solve the Hubbard model within the dynamical cluster approximation (DCA) (Ref. 14) on a $N_{c}$-site cluster. The DCA is a cluster mean-field theory which maps the original lattice model onto a periodic cluster of size $N_{c}=L_{c}^{2}$ embedded in a self-consistent host. Spatial correlations up to a range $L_{c}$ are treated explicitly while those at longer length scales are described at the mean-field level. However the correlations in time, essential for quantum criticality, are treated explicitly for all cluster sizes. To solve the cluster problem, we use weak-coupling expansion continuous time quantum Monte Carlo (QMC) method ${ }^{15,16}$ with highly optimized blocked and delayed updates, ${ }^{17}$ a determinant QMC method which scales linearly in the inverse temperature, ${ }^{18}$ as well as Hirsch-Fye QMC. ${ }^{19,20}$ The fast determinant QMC was used to obtain a converged DCA solution, Hirsch-Fye QMC was used to calculate lattice susceptibilities, and continuous time QMC was used as a control for systematic error. The unit of energy is $t$ in the entire paper.

To make contact with previous results, we perform simulations with $U=6$, but we find that the phase separation becomes more prevalent for $U=8$ for which we present most of our results. We calculate the filling, $n$, versus $\mu$ and the compressibility, $d n / d \mu$, by taking the numerical derivative. We also calculate various susceptibilities including the charge $\chi_{c}(\mathbf{Q}=0, T)$, spin and pairing susceptibilities by solving the lattice Bethe Salpeter equation using the renormalized DCA vertices. ${ }^{20}$ Note that as a consequence of the fluctuationdissipation theorem, $\chi_{c}(\mathbf{Q}=0, T)$ is identical to $d n / d \mu$, but we keep both terms, compressibility and susceptibility, to identify the method by which they are calculated.

\section{RESULTS}

In Fig. 2(a), we plot $n$ versus $\mu$ for $U=6, T=0.077$ and different values of $t^{\prime}$, ranging from 0.0 to 0.4 , on a 16 -site cluster. The filling increases monotonically with the chemical potential and shows a pronounced flat region, associated with the Mott gap, especially for $t^{\prime}<0.4$. The most interesting feature of $n(\mu)$ is an inflection apparent at finite doping, which becomes more pronounced for larger values of $t^{\prime}$. The inflection in $n(\mu)$ translates into a peak in the compressibility (shown on the right axis). The peak becomes sharper and moves closer to half filling as $t^{\prime}$ increases. For $t^{\prime}>0.3$, the peak disappears, as does the plateau in $n(\mu)$ near half filling, associated with the gap. The value of the critical filling at the peak, $n_{c}$, versus $t^{\prime}$ is plotted in the inset. Note that for $t^{\prime}$ $=0, n_{c}=0.86$ agrees with the filling 0.85 of the QCP identified previously for these parameters ${ }^{21,22}$ and a critical filling separating two Fermi-liquid (FL) regions in a closely related $t$ - $J$ model. ${ }^{23}$ These results suggest that the QCP may be associated with charge fluctuations.

To explore this association, we study the behavior of the bulk charge susceptibility, $\chi_{c}(\mathbf{Q}=0, T)$ and its divergence as
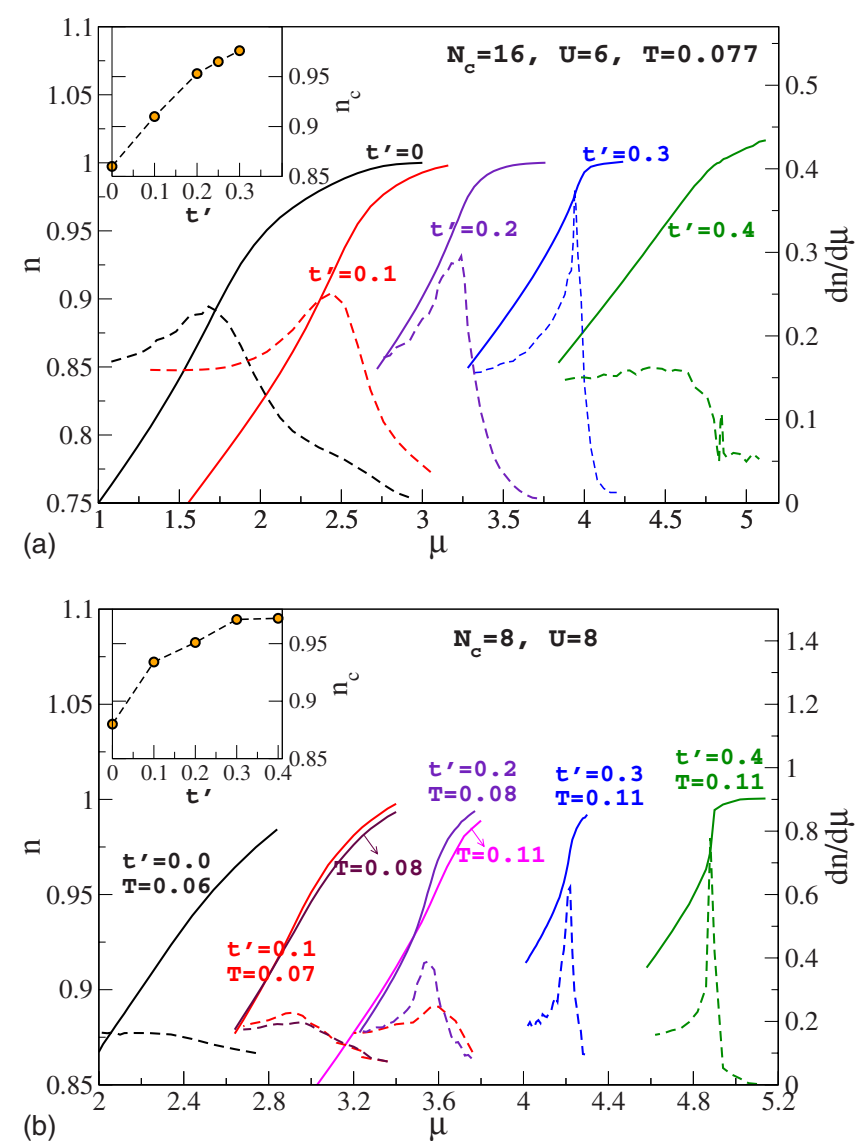

FIG. 2. (Color online) Filling, $n$ (solid lines), and compressibility, $d n / d \mu$ (dashed lines), plotted vs chemical potential, $\mu$, for various values of $t^{\prime}$ for (a) $U=6, N_{c}=16$, and $T=0.077$, and (b) $U=8$, $N_{c}=8$ at different temperatures. The unit of energy is $t$ in all figures. The critical filling, where the compressibility peaks, is plotted in the corresponding inset. In (a) when $t^{\prime} \rightarrow 0$ the peak in the charge susceptibility is located at the QCP identified previously (Ref. 21).

$t^{\prime} \rightarrow 0$. Unfortunately, for this cluster and parameters, the minus sign problem ${ }^{24}$ limits our ability to access temperatures low enough to see a divergence in the charge susceptibility. The minus sign problem becomes worse when the cluster size, $U$ or $\left|t^{\prime}\right|$ increases or the temperature decreases. However, the cluster size, interaction $U$ and $t^{\prime}$ affect the phase diagram in different ways. In previous studies, ${ }^{11}$ we found that for $t^{\prime}=0.3$, clusters with $N_{c}=8,12$, and 16 have roughly the same phase-separation transition temperature when $U$ $=8$. Here, using the same interaction strength, we find that the cluster size effects are stronger for smaller values of $t^{\prime}$. As the cluster size is decreased, the charge susceptibility is somewhat suppressed, and more significantly, the critical doping moves toward half filling. With increasing $U$ in the range from $U=4$ to 8 , the peak in the charge susceptibility moves to lower fillings and higher temperatures. So, despite the worse minus sign problem associated with larger values of the interaction, the region of divergent charge fluctuations becomes larger and more accessible for $U=8$. For this reason, from this point on we will use a smaller cluster with $N_{c}=8$ and $U=8$, in order to access the second-order critical points and investigate their relationship to superconductivity. 


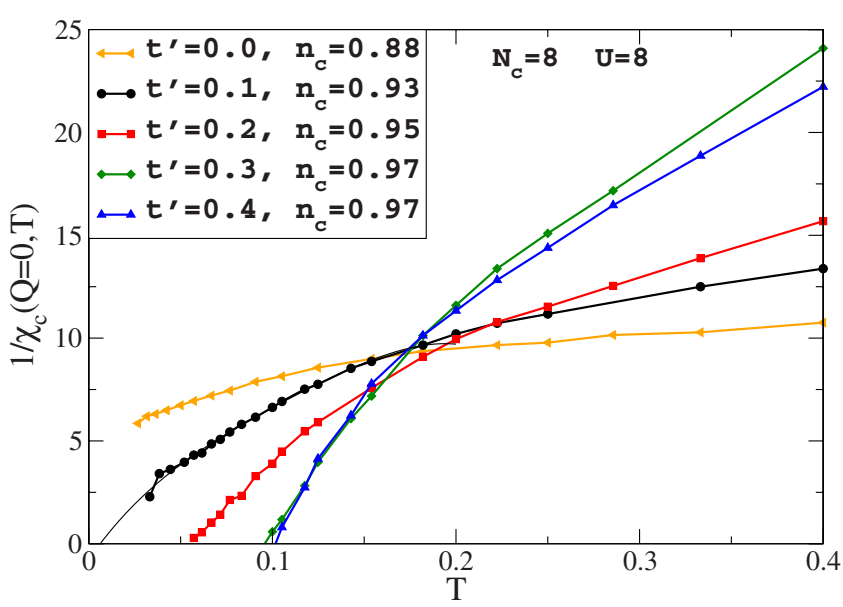

FIG. 3. (Color online) Inverse bulk charge susceptibility vs temperature when $U=8, N_{c}=8$ for several values of $t^{\prime}$. The values of the critical filling $n_{c}$ shown in the legend correspond to the maximum of low-temperature compressibility, or the filling where it first diverges.

In Fig. 2(b), we plot $n$ and $d n / d \mu$ versus $\mu$ for several $t^{\prime}$ and temperatures. Similar to Fig. 2(a), we see a cusp emerge in the compressibility. As the temperature is lowered, the peak in the compressibility is enhanced for $t^{\prime}=0.1$ and 0.2 . However, for a larger $t^{\prime}$, e.g., $t^{\prime}=0.3$, and for $T<0.1$, we find hysteresis between two stable solutions with different values of $n$ for the same value of $\mu .{ }^{11}$ The presence of hysteresis indicates that the system has undergone a first-order phaseseparation transition.

We explore the line of second-order critical points of these first-order transitions as $t^{\prime}$ changes using the charge susceptibility as shown in Fig. 3. Here, the inverse charge susceptibility at $n_{c}$ is plotted versus temperature for different values of $t^{\prime}$ when $U=8$ and $N_{c}=8$. The critical filling identified in the legend is determined as the filling where the compressibility either diverges or is peaked at the lowest accessible temperatures. We find that the temperature of the second-order critical point increases with increasing $t^{\prime}$ and that it moves toward half filling. However, unlike the $U=6$ results shown in Fig. 2(a), the critical point appears to avoid half filling even for $t^{\prime}=0.4$. The stronger Coulomb interaction $U=8$ also strengthens the Mott gap for this $t^{\prime}$ as can be seen in the persistence of the flat region in $n(\mu)$ near $n=1$ for $t^{\prime}=0.4$ [Fig. 2(b)].

The charge fluctuations associated with phase-separation influence the SC phase diagram. This is shown in Fig. 4 for $N_{c}=8, U=8$, and $t^{\prime}=0.0,0.1$, and 0.3 . The pseudogap temperature, $T^{*}$, obtained as the temperature where the bulk spin susceptibility peaks (see Ref. 21), is also plotted. For $t^{\prime}=0$, $T^{*}$ vanishes at the QCP, which for this smaller cluster has moved to $n_{c}=0.88$. Note that for $t^{\prime}=0$, the SC dome is centered on the QCP, suggesting that superconductivity is associated with the quantum fluctuations. For $t^{\prime}=0.1$ and $t^{\prime}$ $=0.3$, the SC dome contains the point where $T^{*} \rightarrow 0$, but is not centered around $n_{c}$. Instead, the second-order point is found on the low-doping side of the SC dome. Note that the maximum SC transition temperature increases slightly with

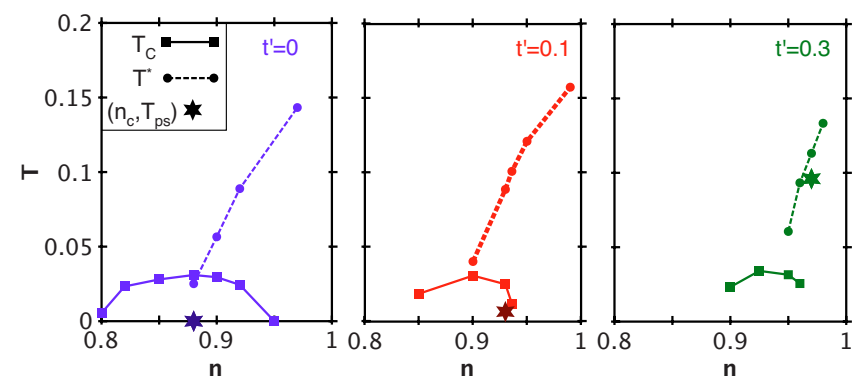

FIG. 4. (Color online) SC transition temperature $T_{c}$ (solid lines), pseudogap temperature $T^{*}$ (dotted lines), and critical points (stars) for the 2D Hubbard model with $U=8, N_{c}=8$, and $t^{\prime}=0,0.1$, and 0.3 . For all $t^{\prime}$ the $T^{*}$ line terminates inside the SC dome. For $t^{\prime}=0$, this termination point coincides with the QCP of the phase-separation transition (star in the left panel). For $t^{\prime}=0.1$ and 0.3 the secondorder critical point is at critical filling $n_{c}=0.93$ and 0.97 , respectively, which is above the SC optimal filling.

increasing $t^{\prime}$ which is in agreement with previous results for a four-site cluster, and the same interaction strength. ${ }^{25}$

\section{DISCUSSION}

A detailed study of the phase diagram of the 2D Hubbard model with next-near-neighbor hopping has allowed us to identify the nature of the QCP under the SC dome. We argue that QCP is the terminal point of a line of second-order critical points associated with first-order phase-separation transitions. The critical temperature is driven to zero as $t^{\prime} \rightarrow 0$. For positive $t^{\prime}$, a Mott liquid and a Mott gas coexist at fixed $\mu .{ }^{11}$ In real materials other parameters, such as electron-phonon interaction, intersite electron-electron interaction or inhomogeneities, might play a role similar to $t^{\prime}$.

It is generally accepted that the model describes the electron-doped cuprates for $t^{\prime}>0$ and the hole-doped cuprates for $t^{\prime}<0$. We find that the model for $t^{\prime}>0$ does not display quantum criticality, but rather classical criticality. The QCP is found only for $t^{\prime}=0$ and as is known from other quantum critical systems, it will strongly affect the system for a wide range of parameters and temperatures around this point, including $t^{\prime}<0$, the model for the hole-doped cuprates.

The relationship of the superconductivity with the QCP at $t^{\prime}=0$ is not yet clear, but the fact that the dome is centered at the QCP suggests that the incipient phase separation creates conditions favorable for superconductivity. ${ }^{26}$ The Mott liquid phase may provide regions where the spin-mediated pairing interaction is strong and the Mott gas may provide regions where there are quasiparticles to pair. It may also be that the incipient charge fluctuations when combined with the antiferromagnetic spin fluctuations enhance the pairing within a narrow region near the QCP. ${ }^{27}$ The phase-separated region might also be related to the pervasive inhomogeneities observed in cuprates which led to theoretical scenarios for an inhomogeneity-based pairing mechanism ${ }^{28,29}$ or an enhancement of pairing interactions. ${ }^{30-34}$ Finally, it has been suggested that in the vicinity of the QCP, even a weak retarded attractive interaction may become far more effective at in- 
ducing pairing. ${ }^{35}$ The relation between the QCP and superconductivity will be explored in greater detail in future studies.

\section{CONCLUSION}

We find that the QCP at $t^{\prime}=0$ of the 2D Hubbard model is the zero-temperature limit of a line of second-order critical points associated with a first-order phase-separation transition, which occur at finite temperature when $t^{\prime}>0$. The filling associated with the second-order critical point is determined from the peak position in the compressibility versus chemical potential. The peak grows by decreasing the temperature or increasing the cluster size or the interaction strength. We also show that for $t^{\prime}>0$ and at $n=n_{c}$, the charge susceptibility diverges at a finite temperature which decreases by decreasing $t^{\prime}$. As $t^{\prime} \rightarrow 0, n_{c}$ moves continuously from values close to half filling to the filling that corresponds to the QCP. For $t^{\prime}=0$, the $\mathrm{SC}$ dome is centered at the QCP where the pseudogap temperature, $T^{*}$, also vanishes. This suggests that the incipient phase separation might play a role in the pairing mechanism. However, for $t^{\prime}>0$, while $T^{*}$ seems to vanish roughly at the center of the dome, phase separation happens at temperatures much higher than the SC temperature with classical critical points that move to the lower-doping side of the dome.

\section{ACKNOWLEDGMENTS}

We would like to thank P. Phillips, S. Kivelson, C. Varma, and J. Zaanen for useful conversations. This research was supported by NSF under Grant No. DMR-0706379. J.M. and M.J. are supported by the NSF PIRE under Project No. OISE-0730290. M.J. and R.T.S. are also supported by DOE SciDAC under Project No. DE-FC02-06ER25792. This research used resources of the National Center for Computational Sciences at Oak Ridge National Laboratory, which is supported by the Office of Science of the U.S. Department of Energy under Contract No. DE-AC05-00OR22725.
${ }^{1}$ P. Coleman and A. J. Schofield, Nature (London) 433, 226 (2005).

${ }^{2}$ S. Sachdev, Nat. Phys. 4, 173 (2008).

${ }^{3}$ A. Kopp and S. Chakravarty, Nat. Phys. 1, 53 (2005).

${ }^{4}$ D. van der Marel, H. J. A. Molegraaf, J. Zaanen, Z. Nussinov, F. Carbone, A. Damascelli, H. Eisaki, M. Greven, P. H. Kes, and M. Li, Nature (London) 425, 271 (2003).

${ }^{5}$ R. Daou, N. Doiron-Leyraud, D. LeBoeuf, S. Y. Li, F. Laliberté, O. Cyr-Choiniére, Y. J. Jo, L. Balicas, J.-Q. Yan, J.-S. Zhou, J. B. Goodenough, and Louis Taillefer, Nat. Phys. 5, 31 (2009).

${ }^{6}$ F. F. Balakirev, J. B. Betts, A. Migliori, I. Tsukada, Y. Ando, and G. S. Boebinger, Phys. Rev. Lett. 102, 017004 (2009).

${ }^{7}$ C. M. Varma, Z. Nussinov, and W. van Saarloos, Phys. Rep. 361, 267 (2002).

${ }^{8}$ D. M. Broun, Nat. Phys. 4, 170 (2008).

${ }^{9}$ M. Vojta, Rep. Prog. Phys. 66, 2069 (2003).

${ }^{10}$ S. A. Grigera, R. S. Perry, A. J. Schofield, M. Chiao, S. R. Julian, G. G. Lonzarich, S. I. Ikeda, Y. Maeno, A. J. Millis, and A. P. Mackenzie, Science 294, 329 (2001).

${ }^{11}$ A. Macridin, M. Jarrell, and Th. Maier, Phys. Rev. B 74, 085104 (2006).

${ }^{12}$ M. Aichhorn, E. Arrigoni, M. Potthoff, and W. Hanke, Phys. Rev. B 76, 224509 (2007).

${ }^{13}$ E. Gull, O. Parcollet, P. Werner, and A. J. Millis, Phys. Rev. B 80, 245102 (2009).

${ }^{14}$ M. H. Hettler, A. N. Tahvildar-Zadeh, M. Jarrell, T. Pruschke, and H. R. Krishnamurthy, Phys. Rev. B 58, R7475 (1998); M. H. Hettler, M. Mukherjee, M. Jarrell, and H. R. Krishnamurthy, ibid. 61, 12739 (2000).

${ }^{15}$ S. M. A. Rombouts, K. Heyde, and N. Jachowicz, Phys. Rev. Lett. 82, 4155 (1999).

${ }^{16}$ A. N. Rubtsov, V. V. Savkin, and A. I. Lichtenstein, Phys. Rev. B 72, 035122 (2005).

${ }^{17}$ K. Mikelson, A. Macridin, E. D’Azevedo, K. Tomko, and M. Jarrell (unpublished).
${ }^{18}$ E. Khatami, C. R. Lee, Z. J. Bai, R. T. Scalettar, and M. Jarrell, Phys. Rev. E 81, 056703 (2010).

${ }^{19}$ J. E. Hirsch and R. M. Fye, Phys. Rev. Lett. 56, 2521 (1986).

${ }^{20}$ M. Jarrell, Th. Maier, C. Huscroft, and S. Moukouri, Phys. Rev. B 64, 195130 (2001).

${ }^{21}$ N. S. Vidhyadhiraja, A. Macridin, C. Sen, M. Jarrell, and M. Ma, Phys. Rev. Lett. 102, 206407 (2009).

${ }^{22}$ K. Mikelsons, E. Khatami, D. Galanakis, A. Macridin, J. Moreno, and M. Jarrell, Phys. Rev. B 80, 140505(R) (2009).

${ }^{23}$ K. Haule and G. Kotliar, Phys. Rev. B 76, 092503 (2007).

${ }^{24}$ E. Y. Loh, J. E. Gubernatis, R. T. Scalettar, S. R. White, D. J. Scalapino, and R. L. Sugar, Phys. Rev. B 41, 9301 (1990).

${ }^{25}$ E. Khatami, A. Macridin, and M. Jarrell, Phys. Rev. B 78, 060502(R) (2008).

${ }^{26}$ S. A. Kivelson and V. J. Emery, in Strongly Correlated Electronic Materials: The Los Alamos Symposium 1993, edited by K. S. Bedell, Z. Wang, and D. E. Meltzer (Addison-Wesley, Redwood City, 1994).

${ }^{27}$ It has been proposed that incommensurate charge fluctuations can mediate $d$-wave pairing, see C. Castellani, C. Di Castro, and M. Grilli, Z. Phys. B: Condens. Matter 103, 137 (1997).

${ }^{28}$ E. Arrigoni, E. Fradkin, and S. A. Kivelson, Phys. Rev. B 69, 214519 (2004).

${ }^{29}$ E. W. Carlson, V. J. Emery, S. A. Kivelson, and D. Orgad, in The Physics of Superconductors, edited by K. H. Bennemann and J. B. Ketterson (Springer-Verlag, Berlin, 2004), Vol. II.

${ }^{30}$ I. Martin, D. Podolsky, and S. A. Kivelson, Phys. Rev. B 72, 060502(R) (2005).

${ }^{31}$ K. Aryanpour, E. R. Dagotto, M. Mayr, T. Paiva, W. E. Pickett, and R. T. Scalettar, Phys. Rev. B 73, 104518 (2006).

${ }^{32}$ W.-F. Tsai and S. A. Kivelson, Phys. Rev. B 73, 214510 (2006).

${ }^{33}$ Y. L. Loh and E. W. Carlson, Phys. Rev. B 75, 132506 (2007).

${ }^{34}$ T. A. Maier, G. Alvarez, M. Summers, and T. C. Schulthess, arXiv:0912.2092 (unpublished).

${ }^{35}$ J. H. She and J. Zaanen, Phys. Rev. B 80, 184518 (2009). 